H. Heise
Regina Zimmermann

\title{
Eine Hundertjährige aus dem Blickwinkel von Künstlern
}

\author{
A Century of Dermatological Clinic from the Point of View of Artists
}

\section{Zusammenfassung}

Die 1902 als eine der ersten deutschen Hautkliniken gegründete Klinik an der Universität Rostock war auch Gegenstand des Interesses von Künstlern. In dieser Abhandlung werden bauliche Aspekte der Klinik und ihre künstlerische Umsetzung betrachtet. Die künstlerisch wertvolle Moulagensammlung der Klinik wurde durch Kriegseinwirkungen leider weitgehend zerstört. Die Atmosphäre in der Klinik reflektierte ein „Künstler-Patient“ mit grafischen Darstellungen.

\section{Abstract}

The dermatological clinic of the University of Rostock was founded as one of Germany's first, in 1902. This paper deals with historical architectural features of the hospital and its artistical realization. The valuable wax moulages of the clinic were destroyed during World War II. An artist, who spent some time in the clinic as a patient, reflected the atmosphere in the hospital with his graphic illustrations.

\section{Einleitung}

Zur Zeit der Wende zum 20. Jahrhundert waren an vielen deutschen Universitäten Bestrebungen im Gange, selbständige Polikliniken und Kliniken der Dermatologie zu errichten. Einige Vorläufer hatten sich bereits Ende des 19. Jahrhunderts, zum Beispiel in Berlin, Breslau, München und Würzburg etabliert [1]. Das mecklenburgische Ministerium in Schwerin tat sich jedoch auf den Rostocker Antrag von 1900 recht schwer, indem erst nach langem Tauziehen am 21.1.1902 die Genehmigung zur Errichtung einer Poliklinik für Haut- und Geschlechtskrankheiten als Universitätsinstitut unter der Leitung eines außerordentlichen Professors für Dermatologie erteilt wurde [2]. Der Lehrstuhl, der neunte in Deutschland, wurde mit dem bekannten Kliniker und Forscher Maximilian Wolters aus Bonn besetzt. Die erste am 24.4.1902 [2] übergebene Hautklinik war zunächst in der räumlichen und materiellen Ausstattung recht dürftig. Wolters gelang es aber, innerhalb kurzer Zeit die Bedingungen deutlich zu verbessern und die Klinik räumlich zu erweitern.
Im Laufe der nun vergangenen hundert Jahre hatte die Klinik bis heute mit großen baulichen Schwierigkeiten zu kämpfen. Es gelang Wolters und der medizinischen Fakultät, den Neubau einer Hautklinik (Abb.1), die am 1.10.1908 eingeweiht wurde, zu erwirken. Dieser Bau war durchaus architektonisch ansprechend im für Kliniken typischen Zeitstil errichtet worden. Mit der Erweiterung 1926 und 1930 durch Professor Frieboes verfügte Rostock über eine für die damalige Zeit recht moderne Hautklinik. Doch der Zweite Weltkrieg, durch den die Klinik und Poliklinik am 24.4.1942 vollständig zerstört wurde, bedeutete zunächst einmal das Ende der Klinik und den Beginn von Provisorien. Der vorläufige Höhepunkt in der provisorischen Einrichtung einer Hautklinik bis zu einem geplanten, aber nie realisierten Neubau war dann am 28. 5.1954 die Übergabe eines Poliklinikneubaus in einer Kriegslücke zwischen den als Bettenstationen dienenden Wohnhäusern in der Augustenstraße. Dieser Standort ist heute noch der Stammsitz der Klinik und Poliklinik, wenn auch die Bettenstationen inzwischen verlagert wurden. 


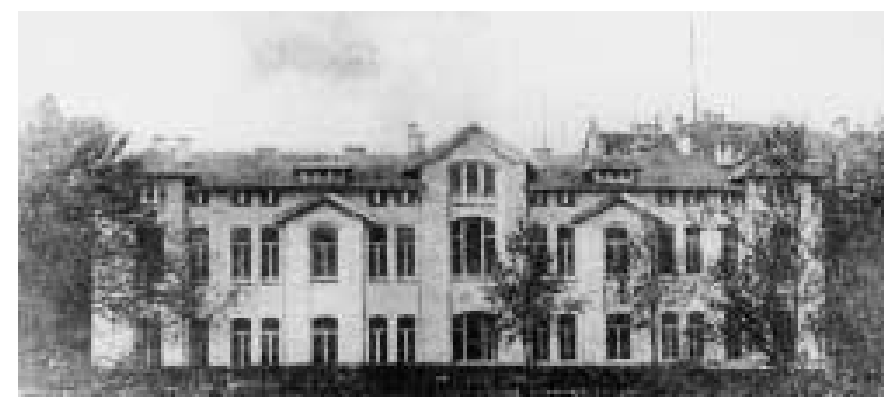

Abb. 1 Die erste, später erweiterte und im Krieg zerstörte Universitäts-Hautklinik in Rostock. ble auf, obgleich aus ökonomischen Gründen wesentliche Änderungen nicht vorgenommen werden konnten. Durch die etwa gleiche Höhe der Seitengebäude und durch die Anpassung des Neubaus an das links sich anschließende alte Gebäude entstand scheinbar ein einheitliches Ensemble, das durch den Eingangsbereich eine stark betonte Mittelgliederung erhielt. Neben der sitzer inzwischen rekonstruiert wurde und nunmehr als CaritasZentrum dient. Die noch von der Hautklinik genutzten Gebäude bedürfen äußerlich auch einer dringenden Renovierung, was aber in Anbetracht einer geplanten Aufgabe des Standortes Augustenstraße in dieser Weise nicht realisiert werden wird.

Fotografische Detailaufnahmen (Abb.3) beziehen sich auf den architektonisch am besten gelungenen Mittelteil, der durch den Anbau eines behindertengerechten Zuganges jedoch heute etwas von seiner Harmonie verloren hat.

Im Gegensatz dazu bietet die Rückfront der Klinik (Abb. 4) bis auf ein über drei Etagen reichendes Treppenhausfenster keine interessanten Aspekte. Hier vermitteln die baulichen Bedingungen einen eher ungeordneten Eindruck. Gleiches trifft auch für die Umgebung der Klinik zu, die durch Altbauten und neue Lückenbzw. Abrissbebauungen geprägt ist.

Von besonderem Interesse ist aber, dass gerade diese scheinbar bauliche und räumliche Unordnung Maler und Grafiker besonders inspirierte, von denen uns jedoch keine Darstellungen der attraktiveren Vorderfront vorliegen.

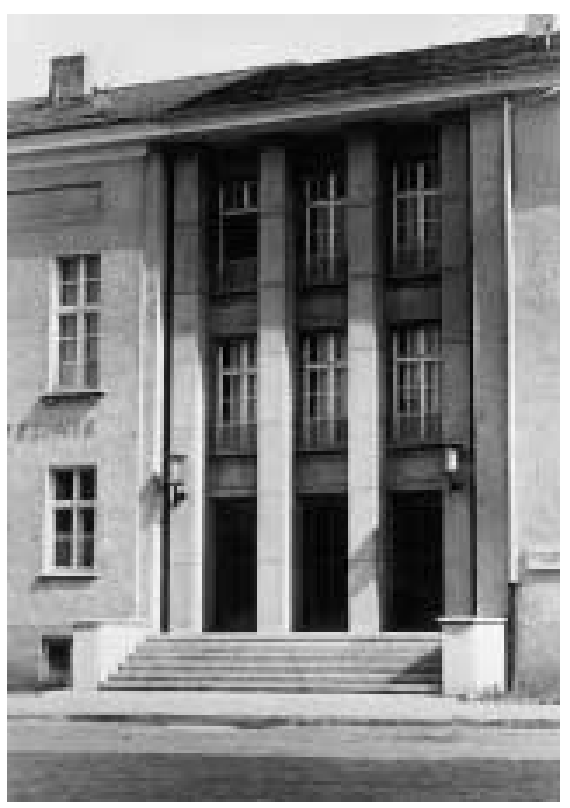

Abb. 3 Detailaufnahme des schlicht und sachlich gestalteten Eingangsbereiches. gelungenen Einbeziehung der seitlichen Wohnhäuser fällt die im architektonischen Stil der 50er Jahre des vergangenen Jahrhunderts gut und funktional gestaltete Fassade auf (entwerfender Architekt: Prof. Matthias Schubert, geb. 1928). Die Schaffung dieses einheitlichen äußeren Bildes ist umso erstaunlicher, als im Inneren vier verschiedene Etagenniveaus bestehen, die über Treppen verbunden sind und der dreigeschossige Neubau mit dem zweigeschossigen alten Wohnhaus verbunden werden musste.

Dieser einheitliche Aspekt ist heute etwas verlorengegangen, da das rechts außen stehende, früher nur angemietete Gebäude, wegen Baufälligkeit nicht mehr genutzt werden konnte, vom Be-

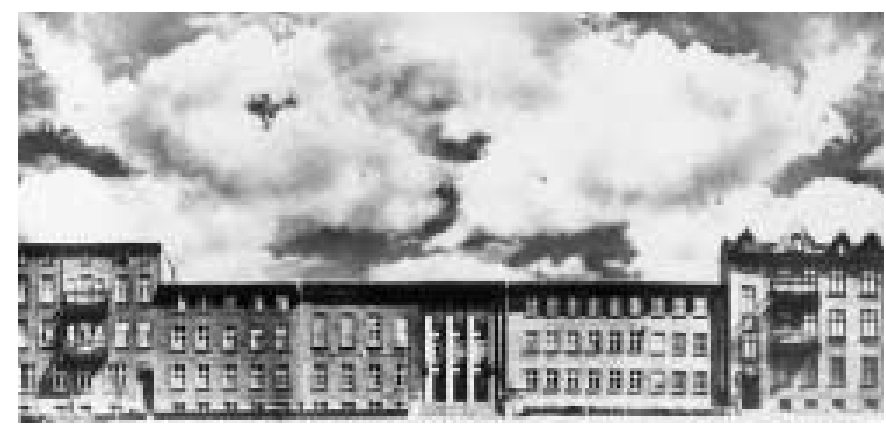

Abb. 2 Fotomontage einer Gesamtansicht der Anfang der 50er Jahre des vergangenen Jahrhunderts durch Lückenbebauung geschaffenen Universitäts-Klinik und Poliklinik für Hautkrankheiten.

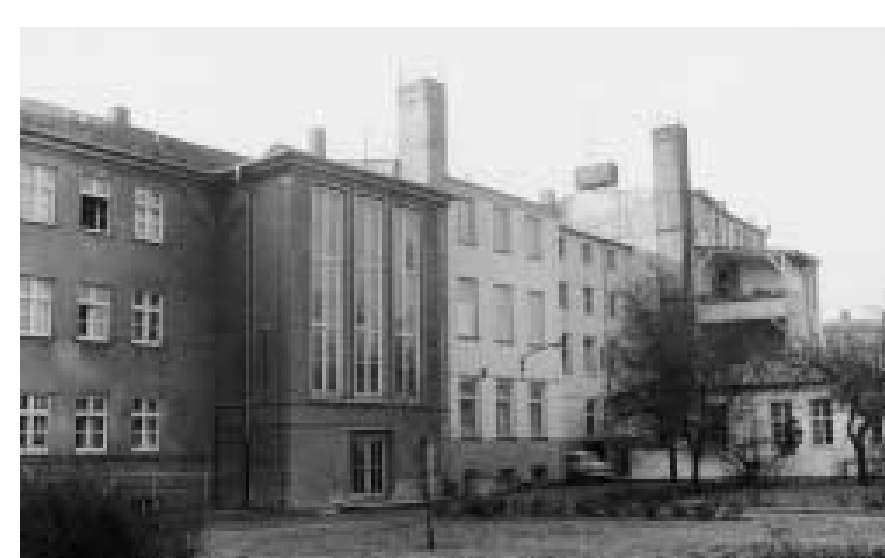

Abb. 4 Die Rückfront offenbart das Einbeziehen unterschiedlicher Häuser aus verschiedenen Bauepochen. Die beiden rechten Häuser stammen aus der Erstbebauung des Stadtteils gegen Ende des 19. Jahrhunderts. 
Zur Veranschaulichung sollen zwei Beispiele künstlerischer Umsetzung herausgegriffen werden.

Aus Anlass des 60. Geburtstages des damaligen Klinikdirektors Prof. Heinz Flegel im Jahre 1983 hatten die Assistenten der Klinik den in Rostock bekannten Wissenschaftler und Maler Lothar Kalbe (Dr. rer. nat. Lothar Kalbe, geb. 12. 2.1927, wissenschaftlicher Assistent am Institut für allgemeine Hygiene von 1957-1992) gebeten, ein Bild von der Klinik zu malen. Das dann fertiggestellte Werk (Abb.5) verblüffte allgemein, indem es den baulich chaotischsten Bereich der Klinik beinhaltete. Unter besonderer Betonung eines ohnehin störenden Schornsteins (siehe aktuelles Foto, Abb. 6) sind hier von der Rückseite die Gebäude aus den verschiedensten Bauepochen dargestellt. Einmal handelt es sich um das Eckwohnhaus aus dem Ende des 19. Jahrhunderts mit dem sich anschließenden ebenfalls rekonstruierten Gebäude, zum anderen um den 1952/53 erweiterten Flachbau des Hörsaals. Dieses Bild ist eigentlich recht dazu angetan, den provisorischen Charakter der Verschmelzung unterschiedlicher Häuser als Notlösung für den weiteren Klinik- und Lehrbetrieb zu demonstrieren.

Noch auffallender ist die spontane Umsetzung der Eindrücke im Bereich der Klinik durch den bekannten Illustrator Werner Schinko während seines Aufenthaltes in der Klinik im Jahre 1976/77. Der 1929 geborene Werner Schinko, der 1951 bis 1955 an der Hochschule für bildende und angewandte Kunst in BerlinWeißensee studierte und seit vielen Jahrzehnten in Röbel in Mecklenburg sesshaft ist, gehört zu den gegenwärtig führenden Buchgrafikern und Illustratoren. Die Gebäude der Klinik müssen den Künstler so wenig inspiriert haben, dass sich unter den vie-

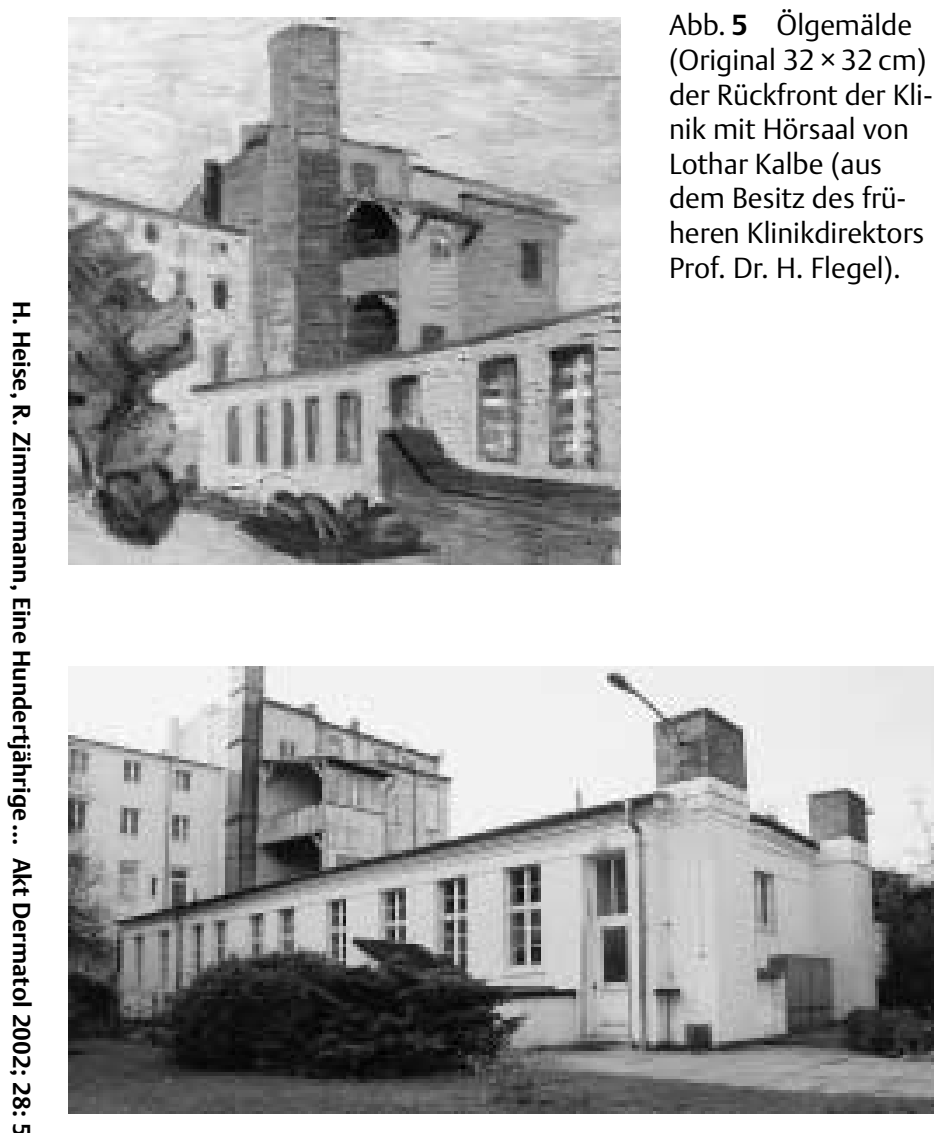

G Abb. 6 Fotografische Aufnahme des aktuellen Zustandes; im Hinterज grund das ursprüngliche Wohnhaus aus dem 19. Jahrhundert.

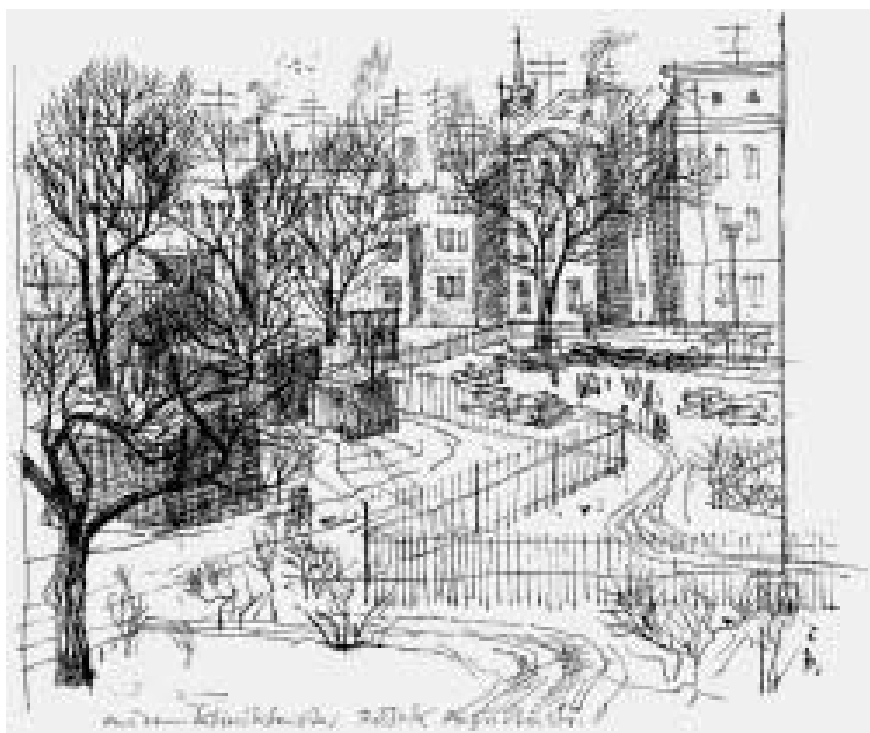

Abb. 7 Zeichnung aus dem Skizzenbuch des Grafikers Werner Schinko aus dem Jahre 1976 vom Gartenbereich der Klinik.

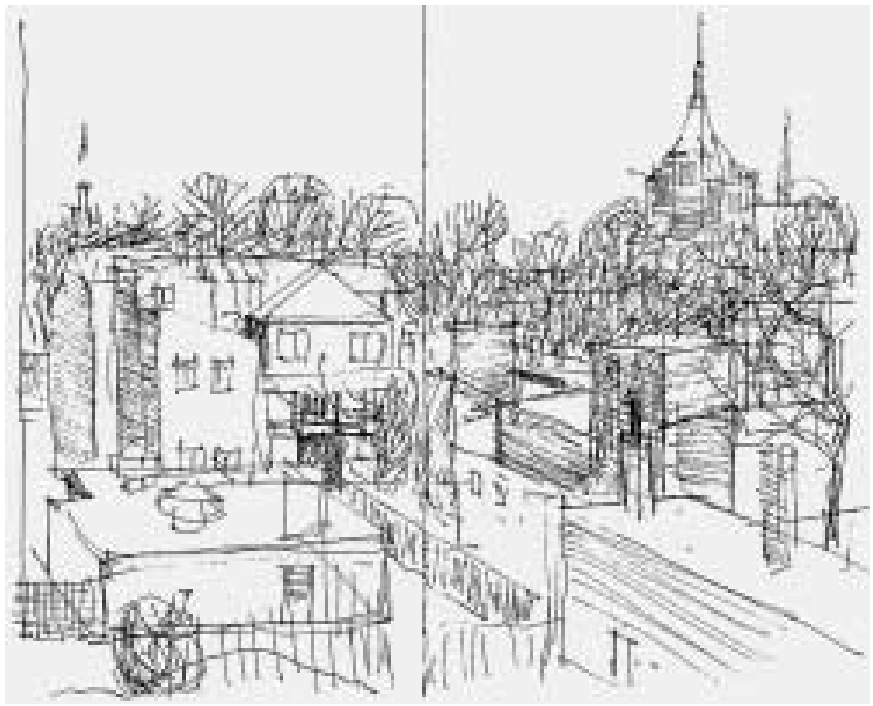

Abb. 8 wie Abb. 7, Blick auf die Marienkirche.

len angefertigten Zeichnungen keine befindet, die eine Ansicht der Klinik selbst zeigt. Die Darstellungen von Werner Schinko lassen dagegen den ungeordneten baulichen Zustand im Umfeld der Klinik erkennen (Abb. 7 u. 8). Daneben zeigt er aber auch im Blick aus dem Fenster (Abb.9) das 24-geschossige Wohnhochhaus, das Ende 1969 praktisch im Garten der Klinik unter Abriss von Nebengebäuden entstanden war und das das erste Gebäude des geplanten sozialistischen Rostocker Stadtzentrums sein sollte. Der für die Klinik bedrückende Aspekt lässt sich anhand dieser Zeichnung sehr gut nachempfinden. Der Zustand der Klinik selbst hat den Künstler offensichtlich nicht zu weiteren Darstellungen animiert.

\section{Klinilkimpressionen eines Küinstlers}

Werner Schinko verdanken wir aber auch eine Reihe von Skizzenblättern vom Stationsalltag, die durchaus die Sicht der Patienten wiedergeben. Sicher bedeutete dieses Skizzieren für den 


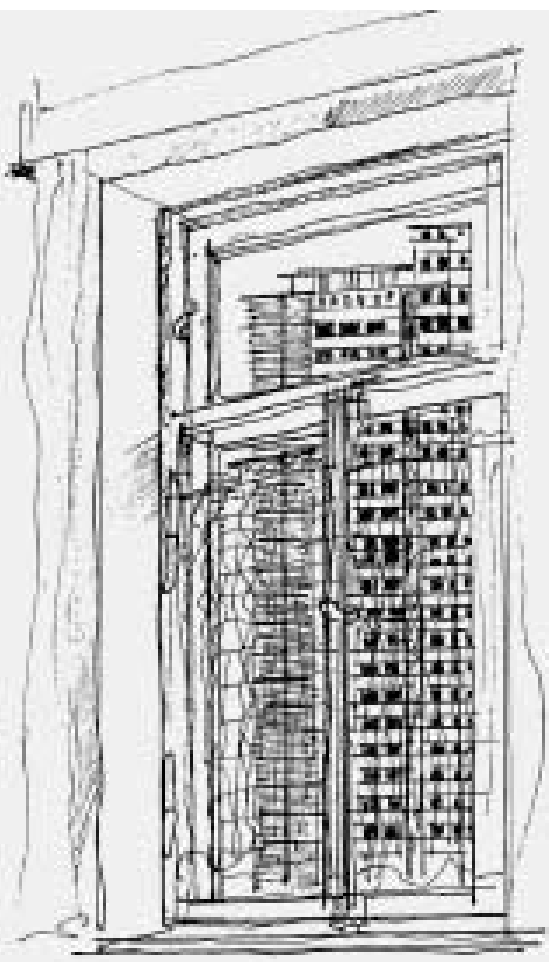

54
Abb. 9 Skizze von W. Schinko mit der Bezeichnung „Mein Fensterplatz“; erdrückender Eindruck eines auf dem Gelände der Klinik neu gebauten Wohnhochhauses.

Abb. 10 Der Mann mit dem Kopfverband; aus dem Skizzenbuch von W. Schinko.

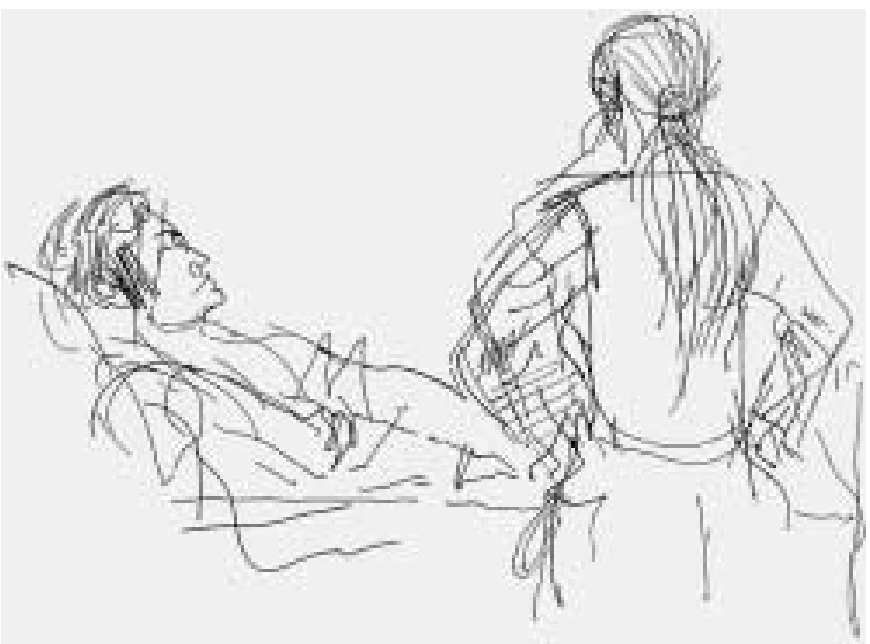

Abb. 11 Ärztin am Krankenbett; Skizze von W. Schinko.

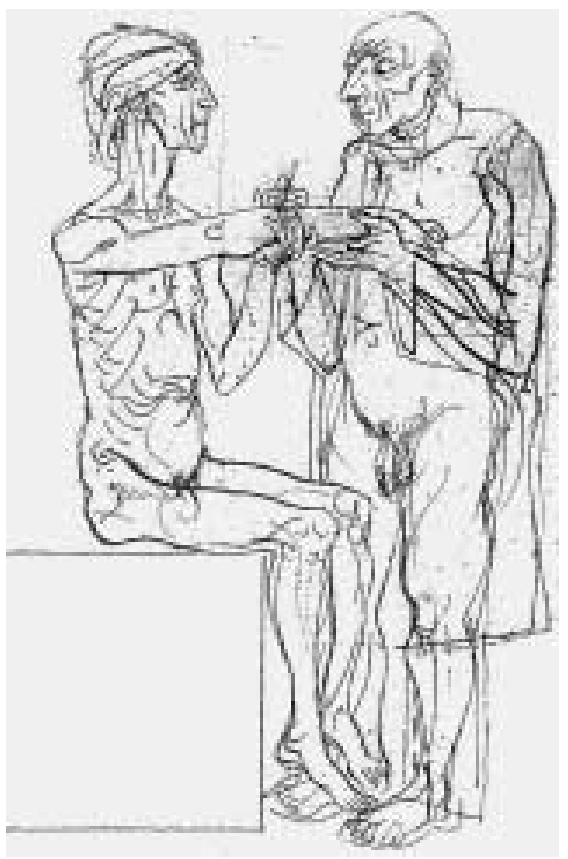

Abb. 12 Die Untersuchung des Hautpatienten; Radierung von W. Schinko.

auch ein gewisses Ausgeliefertsein dem medizinischen Personal gegenüber. Nur so ist die Darstellung der selbstbewussten Haltung der Ärztin am Krankenbett (Abb. 11) oder die des untersuchenden Arztes am Patienten (Abb. 12) zu verstehen. Die in der Dermatologie notwendige Ganzkörperuntersuchung führt aber auch offensichtlich zu gewissen gedanklichen Aversionen, die letztlich auch in der Darstellung von Patient und untersuchendem Arzt ihren Ausdruck finden. Hier wird schließlich gezeigt, dass sich hinter der allmächtigen Respektperson des Arztes unter dem schützenden Kittel auch nur ein Mensch verbirgt (Abb.12). Die oftmals gedrückte Stimmung eines Hautpatienten wird indirekt über den unnahbaren Gesichtsausdruck der behandelnden Ärzte übertragen.

Mittlerweile sind seit der Schaffung dieser Skizzen zwar 25 Jahre, ein Viertel der Gesamtzeit des Klinikjubiläums, vergangen, doch dürfte diese Einstellung der Hautpatienten auch heute im Wesentlichen noch unverändert sein. Hautkrankheiten werden 
von Betroffenen emotional oftmals schwer verarbeitet. In diese Situation muss sich der Hautarzt hineinversetzen.

\section{Die Moulagensammlung}

Die Betrachtung zur Kunst in der Rostocker Universitäts-Hautklinik wäre unvollständig, wenn man nicht auch die historische Moulagensammlung erwähnen würde. Der Grundstein dafür wurde von Professor Wolters mit der Gründung der Klinik gelegt. Durch die Kunstfertigkeit der Moulageure wurden Meisterwerke geschaffen, die für die damalige Zeit einen sehr hohen Stellenwert als Anschauungsmaterial in der Lehre und für Verlaufsbeobachtungen von Hautkrankheiten [4] hatten.

In Rostock lag die Herstellung der Moulagen zuerst in den Händen von Fräulein Auguste Kaltschmidt und später von Herrn Kurt Krug. Diese beiden schufen mit enormem Fleiß schließlich eine Sammlung von etwa 3000 Moulagen [5]. Leider wurde diese Sammlung im Zweiten Weltkrieg 1942 mit der Klinik fast vollständig zerstört. Lediglich 34 der künstlerisch wertvollen Moulagen konnten bis zum heutigen Zeitpunkt gerettet werden [6]. Aus ihnen lassen sich einerseits gewisse Wandlungen bei den Krankheitsbildern ablesen, andererseits ist manches Krankheitsbild aus heutiger Sicht neu zu klassifizieren, da inzwischen neue
Krankheiten beschrieben wurden. Es sind aber auch Moulagen von Krankheiten, die heute überhaupt nicht mehr bei uns gesehen werden, wie Rotz und Fleckfieber, darunter. Insgesamt überwiegt unter dem Rostocker Moulagenerbe - wie auch anderswo - die Darstellung syphilitischer Krankheitsbilder.

Die Bewahrung dieses gesamten künstlerischen Erbes sollte für eine Klinik im Sinne einer echten und verbindenden Traditionspflege eine ständige Verpflichtung sein.

\section{Literatur}

${ }^{1}$ Scholz A. Geschichte der Dermatologie in Deutschland. Berlin, Heidelberg, New York: Springer, 1999

${ }^{2}$ Schulze W. Die Dermatologische Klinik der Universität Rostock. Ein Rückblick auf ihre Gründung und Entwicklung. Hautarzt 1955; 6: $84-89$

${ }^{3}$ Ebert G. Das Atelierfest - Betrachtungen über die Kunst zu leben. Halle-Leipzig: Mitteldeutscher Verlag, 1979: 206-207

${ }^{4}$ Ständer S, Crout J, Sunderkötter C, Ständer H. Die Moulagensammlung der Universitäts-Hautklinik Münster. Hautarzt 2001; 52: 662 - 666

${ }^{5}$ Bohnenstengel G. Zur Geschichte der Universitäts-Hautklinik Rostock. Wiss Zschr Univ Rostock Math-Nat Reihe 1972; 21: 9-17

${ }^{6}$ Heise H, Schlecht K, Zimmermann R, Gross G. Die Rostocker Moulagen-Sammlung. Hautarzt 2002; 53: (im Druck)

\section{Buchbesprechung}

\section{Autoimmune Diseases of the Skin. Pathogenesis, Diagnosis, Management}

Michael Hertl (Hrsg.)

373 S. Springer, Wien 2001. Geb. 49,-. ISBN 3-211-83598-9

Solche nehmen zu an Häufigkeit und an Bedeutung, weshalb es nötig ist und verdienstvoll, eine Monographie zu diesem brandaktuellen Thema vorzulegen. Herr Kollege Michael Hertl hat dies mit einer Gruppe von 27 Autoren, alles in ihrem Gebiet welterfahrene und experimentell erprobte Fachleute, unternommen. Es ist meisterlich gelungen, in englischer Sprache, und umfasst 346 Seiten mit einem guten Register. Die Pathogenese steht zum Beginn: die biologisch verschiedenen Mechanismen der Bildung von Autoantikörper und der Toleranzinduktion werden ebenso dargelegt, wie die exakte Charakterisierung der möglichen Autoantigene sowie deren Struktur, Funktion und Verteilung. Daraus lassen sich klinische Morphologie, Anordnung und Verteilung wie auch die funktionellen Beeinträchtigungen derselben ableiten. So kann Verständnis für die enorme Vielfalt der klinischen Symptomatik und die unterschiedliche Verteilung auf ein oder mehrere Organsysteme gewonnen werden. Und fast immer ist das Hautorgan maßgeblich beteiligt. Der Kliniker bekommt manchmal den Eindruck, als ob dieses große und ober- flächliche Organ eine Art Filterfunktion einnimmt, Immunkomplexe fixiert, entzündliche Reaktionen auf sich zieht und so andere, sensiblere und unentbehrlichere Organe zu entlasten versucht. Spielen möglicherweise die speziellen Gefäßanordnungen in der Haut und die niedrigere Oberflächentemperatur eine Rolle dabei? Neben der Pathogenese wird auch ein Kapitel der Epidemiologie und eines den neuen, auch experimentellen Therapien gewidmet. So klammern grundsätzliche Kapitel die zehn klinischen Kapitel ein, die sich jeweils einer distinkten Gruppe von Autoimmunkrankheiten an der Haut (und anderer Organe) zuwendet: blasenbildende Hautkrankheiten, Sklerodermie, Lupus erythematosus, Dermatomyositis, gemischte Bindegewebskrankheit, Sjögren Syndrom, Psoriasis mit Arthropathie, chronische Urtikaria, lichenoide Exantheme und Vaskulitis. Diese Kapitel sind von verschiedenen Autoren verfasst und zeigen doch eine erfreulich einheitliche Gestaltung und profunde Darstellung der Materie, eine bemerkenswerte Leistung des Herausgebers! Das Buch ist eine Bereicherung für Kliniker und Experimentatoren, für niedergelassene Kollegen auch, für Dermatologen und Mediziner aller Couleur.

Ernst G. Jung, Heidelberg 